\section{Padrão de comportamento relacionado às práticas sexuais e ao uso de drogas de adolescentes do sexo feminino residentes em Vitória, Espírito Santo, Brasil, 2002}

\author{
Behavior patterns related to sexual practices \\ and drug use among female adolescents \\ in Vitória, Espírito Santo, Brazil, 2002
}

\author{
1 Núcleo de Doenças \\ Infecciosas, Universidade \\ Federal do Espírito Santo, \\ Vitória, Brasil. \\ 2 Escola Nacional de Saúde \\ Pública, Fundação Oswaldo \\ Cruz, Rio de Janeiro, Brasil. \\ 3 Centro de Informação \\ Científica e Tecnológica, \\ Fundação Oswaldo Cruz, \\ Rio de Janeiro, Brasil. \\ Correspondência \\ A. E. Miranda \\ Núcleo de Doenças \\ Infecciosas, Universidade \\ Federal do Espírito Santo. \\ Av. Marechal Campos 1468, \\ Vitória, ES 29040-090, Brasil. \\ espinosa@ndi.ufes.br
}

\begin{abstract}
Adolescents are a vulnerable group for sexually transmitted diseases (STDs). The goal of this study was to describe behavior patterns among female adolescents (15-19 years) in relation to sexual practices and drug use in an area covered by the Health Family Program in Vitória, capital city of Espírito Santo State, Brazil. A descriptive study from March to June 2002 was performed. A face-to-face interview was conducted and urine samples were collected for the Chlamydia trachomatis LCx test. A total of 464 adolescents were included in the study. Sixty-nine percent were already sexually active; $12.8 \%$ reported previous STDs, $14 \%$ illicit drug use, and 3.7\% a history of prostitution. Only 23.4\% reported regular condom use, although more than $90 \%$ had received information on STD/AIDS risks and prevention. History of pregnancy was reported by $31.6 \%$ of the adolescents, and a previous HIV test by $17 \%$. Although they reported receiving information on STD/AIDS, they were failing to take adequate self-protective measures. The results highlight the need for preventive measures, including STD screening and risk-reduction programs.
\end{abstract}

Adolescent; Sexually Transmitted Diseases; Acquired Immunodeficiency Syndrome; Sexual Behaviour
Angélica Espinosa Miranda 1,2 Angela Maria Jourdan Gadelha 2 Célia Landmann Szwarcwald 3

\section{Introdução}

A adolescência é um conceito moderno que significa o período de vida iniciado na puberdade, e que acaba quando o jovem entra no que, culturalmente, se considera a idade adulta (maturidade social e/ou independência econômica). É durante a fase da adolescência que o indivíduo se desenvolve física e emocionalmente, se inicia sexualmente, e adota comportamentos, influenciado pelo meio sócio-ambiental 1 .

Entretanto, para facilitar os estudos dirigidos, especificamente, à adolescência, a definição proposta pela Organização Mundial da Saúde (OMS) é baseada apenas na faixa etária estabelecendo que adolescente é toda pessoa com idade entre 10 e 19 anos 2. Segundo o Estatuto da Criança e do Adolescente 3 , lei brasileira que dispõe sobre a proteção integral à criança e ao adolescente, adolescente é toda pessoa entre 12 e 18 anos. Recentemente, diversos autores têm recomendado a inclusão do grupo de 20 a 24 anos de idade, pela semelhança no perfil de morbi-mortalidade 4 .

Atualmente, 1,7 bilhões de pessoas (mais do que a quarta parte dos habitantes do planeta) encontram-se na faixa etária de 10 a 24 anos, sendo que $86,0 \%$ dos indivíduos deste grupo etário habitam em países em desenvolvimento 5 . Esta geração atual de jovens é a mais educada e a mais urbana da história. Todavia, ao mesmo tempo em que a urbanização tem aumen- 
tado o acesso à educação e aos serviços de saúde, os adolescentes são mais expostos aos riscos do uso de drogas lícitas e ilícitas, à violência e às infecções de transmissão sexual, incluindo o HIV/AIDS 2.

No que se refere à saúde da adolescente em particular, conforma-se, atualmente, um quadro de saúde em que ganham relevância os aspectos relacionados à sexualidade, em adição àqueles relativos à concepção, à gravidez e ao parto 6 . Entre as jovens do sexo feminino de 15 a 19 anos, as complicações relacionadas à gravidez, ao parto e aos abortos em condições de risco são a principal causa de morte em muitas partes do mundo. Por outro lado, não podemos deixar de citar as taxas elevadas de infecções sexualmente transmissíveis, incluindo a infecção pelo HIV 7 .

Após o advento da infecção pelo HIV, o controle das doenças sexualmente transmissíveis (DST) começou a ser considerado prioritário, pois foi visto que a prevenção e o controle dessas infecções representam oportunidades únicas de melhorar a saúde reprodutiva da mulher 8,9. Tanto na Conferência Internacional sobre População e Desenvolvimento em 1994, como em sua revisão no ano de 1999, os participantes consideraram as adolescentes como um grupo especialmente vulnerável, comprometendo-se a fornecer subsídios para a formação e implementação de serviços em saúde sexual e reprodutiva, de boa qualidade, específicos para adolescentes 5 .

O objetivo deste estudo é descrever o comportamento sexual de risco em relação às infecções sexualmente transmissíveis de adolescentes brasileiras, residentes em uma área de Vitória, capital do Estado do Espírito Santo, assistida pelo Programa Saúde da Família (PSF), com a finalidade de elaborar estratégias de prevenção e assistência direcionadas a esta população.

\section{Metodologia}

Foi realizado um estudo por amostragem nos bairros da região de Maruípe, em Vitória, onde o PSF já estava implantado, e que dispunham de uma lista completa dos domicílios. As micro-regiões incluídas foram: Consolação, Gilson Santos e Andorinhas.

\section{Constituição da amostra}

Adolescentes do sexo feminino, de 15 a 19 anos, que habitavam nas regiões descritas acima. A lista de adolescentes foi obtida através do ca- dastro, feito pelo PSF, de todas as jovens desta faixa etária, que eram residentes nas regiões citadas. Com base neste cadastro, identificaramse 3.367 adolescentes e se procedeu a uma seleção aleatória simples das adolescentes que participaram do estudo.

O tamanho da amostra foi calculado com base na estimação da prevalência da infecção por Chlamydia trachomatis em adolescentes do sexo feminino, com um intervalo de confiança de $95,0 \%$. Dado que no estudo piloto a prevalência observada foi de $11,4 \%$, entre as sexualmente ativas, considerando-se um erro bilateral de 3,0\%, o tamanho da amostra foi calculado em 357 mulheres. Levando-se em consideração que, aproximadamente, 30,0\% das adolescentes nesta faixa etária não têm atividade sexual 10, o tamanho final da amostra foi de 464 adolescentes.

O período de coleta de dados ocorreu em quatro meses, de março a junho de 2002, sendo realizado por cinco entrevistadoras, enfermeiras do PSF, treinadas para tal.

\section{Questionário}

O questionário utilizado foi baseado em outro da ACSJ (Analyse des Comportements Sexuels des Jeunes) 11 e validado no estudo piloto. O instrumento continha dados sócio-demográficos (idade, escolaridade, religião, profissão, estado civil e dados sobre a família); dados clínicos (sintomas DST, contracepção, gravidez e abortos); sexuais (carícias, beijos, relações não-sexuais, primeira relação sexual); sobre comportamentos de risco (uso de preservativos, número de parceiros sexuais, prostituição, uso de álcool e drogas, transfusão de sangue) para infecção pelo HIV e outras DST e conhecimentos sobre contracepção. Todas as perguntas contidas no questionário eram fechadas.

\section{Testes laboratoriais}

Foi coletada uma amostra de $10 \mathrm{ml}$ de urina para realização de testes de biologia molecular LCx, laboratórios ABBOTT para Chlamydia. Os testes LCx usam a tecnologia de amplificação da Reação em Cadeia da Ligase (LCR - ligase chain reaction) no sistema de sonda LCx para detecção direta e qualitativa do plamídio DNA da C. trachomatis e Neisseria gonorrhoea. Pode ser usado para detecção de amostras de secreção uretrais e endocervicais e amostra de primeiro jato de urina. Os testes foram realizados no Núcleo de Doenças Infecciosas da Universidade Federal do Espírito Santo. 
Todas as informações foram codificadas e armazenadas anonimamente em um banco de dados criado para este fim. Foi utilizado o programa estatístico SPSS, versão 9.0.

No presente estudo, foram considerados os seguintes aspectos: dados sócio-demográficos; experiência de violência familiar; comportamento sexual; uso de drogas lícitas e ilícitas; uso e negociação do uso de preservativo masculino; conhecimento sobre formas de transmissão do HIV. Os resultados dos testes laboratoriais, presença de infecções sexualmente transmissíveis e demais aspectos comportamentais serão objeto de estudo posterior.

A análise estatística consistiu na utilização de métodos descritivos e no uso de testes $\chi^{2}$ de associação entre variáveis.

\section{Aspectos éticos}

Este projeto foi submetido e aprovado pelo Comitê de Ética e Pesquisa da Fundação Oswaldo Cruz. Agentes de saúde foram às residências fazer o convite às adolescentes selecionadas e pediram autorização, por escrito, aos pais para que elas participassem do projeto. Os pais foram convidados a comparecer à unidade para esclarecimento de qualquer dúvida ou para maiores explicações. Todas as adolescentes selecionadas foram convidadas a participar do estudo em caráter voluntário. Aquelas que aceitaram participar, assinaram um termo de consentimento escrito após receber as informações sobre o projeto. Elas receberam tratamento, de acordo com as normas do Manual de Controle das Doenças Sexualmente Transmissíveis do Ministério da Saúde, para as infecções diagnosticadas 12 .

\section{Resultados}

Todas as adolescentes selecionadas no processo de amostragem aceitaram participar do estudo. Do total de 464 participantes, apenas três se recusaram a coletar a amostra de urina para realização dos testes diagnósticos, participando somente da entrevista. O índice de resposta foi, portanto, de $99,4 \%$.

As características sócio-demográficas encontram-se descritas na Tabela 1. A média de idade das participantes foi de 17 anos e a distribuição por ano de idade, entre 15 e 19 anos, foi, praticamente, uniforme. A média do número de anos completos de estudo foi de 9 anos, sendo que nenhuma adolescente era analfabe-
Tabela 1

Características sócio-demográficas e de comportamento das adolescentes de 15 a 19 anos, residentes na região de Maruípe, Vitória, Espírito Santo, Brasil.

\begin{tabular}{|c|c|c|}
\hline Variáveis & $\mathbf{n}$ & $\%$ \\
\hline \multicolumn{3}{|l|}{ Idade (anos) } \\
\hline 15 & 99 & 21,3 \\
\hline 16 & 88 & 19,0 \\
\hline 17 & 89 & 19,2 \\
\hline 18 & 87 & 18,8 \\
\hline 19 & 101 & 21,8 \\
\hline \multicolumn{3}{|l|}{ Bairro de origem } \\
\hline Consolação & 192 & 41,4 \\
\hline Andorinhas & 124 & 26,7 \\
\hline Bairro da Penha & 148 & 31,9 \\
\hline \multicolumn{3}{|l|}{ Escolaridade } \\
\hline Ensino fundamental (1a a 4ạ série) & 13 & 2,8 \\
\hline Ensino fundamental (5ạ a 8a série) & 149 & 32,1 \\
\hline Ensino médio (9ạ a 11ạ série) & 275 & 59,3 \\
\hline Ensino superior & 27 & 5,8 \\
\hline \multicolumn{3}{|l|}{ Estado civil } \\
\hline Solteira & 382 & 82,3 \\
\hline Casada/mora com parceiro & 79 & 17,0 \\
\hline Separada/divorciada & 2 & 0,4 \\
\hline Viúva & 1 & 0,2 \\
\hline \multicolumn{3}{|l|}{ Reside com } \\
\hline Pais & 236 & 50,9 \\
\hline Somente com a mãe & 105 & 22,6 \\
\hline Somente com o pai & 9 & 1,9 \\
\hline Avós & 17 & 3,7 \\
\hline Amigos & 9 & 1,9 \\
\hline Namorado/marido & 65 & 14,0 \\
\hline Outros & 23 & 5,0 \\
\hline \multicolumn{3}{|l|}{ Renda familiar (salários mínimos)* } \\
\hline Até 1,9 & 98 & 21,1 \\
\hline $2-2,6$ & 133 & 28,7 \\
\hline $2,7-3,6$ & 109 & 23,5 \\
\hline $3,7-15$ & 124 & 26,7 \\
\hline Têm atividade profissional remunerada & 95 & 20,5 \\
\hline Freqüenta escola & 342 & 73,7 \\
\hline Pratica alguma atividade esportiva semanalmente & 179 & 38,6 \\
\hline Tabagismo atual regular (mais de 5 cigarros ao dia) & 48 & 10,3 \\
\hline Uso regular de álcool (pelo menos uma vez por semana) & 127 & 27,4 \\
\hline Uso de alguma droga ilícita & 42 & 14,0 \\
\hline Possui grupo de amigos & 374 & 80,6 \\
\hline
\end{tabular}

* O salário mínimo na época do estudo era de $\mathrm{R} \$ 200,00$. 
ta e $27,1 \%$ das participantes completaram, pelo menos, o ensino médio. No que diz respeito ao uso regular de bebida alcoólica, $27,4 \%$ das participantes revelaram consumir, pelo menos, uma vez por semana. Em relação ao fumo, 10,3\% fumam regularmente. Quanto às drogas ilícitas, $9,7 \%$ relataram uso de maconha e $1,9 \%$ o uso de drogas injetáveis, sendo que $14,0 \%$ responderam que utilizaram, pelo menos, uma droga ilícita.

Na Tabela 2, observa-se o relato de violência doméstica, analisada segundo o nível de renda familiar. Primeiramente, percebe-se que $36,7 \%$ das adolescentes relataram algum tipo de violência na família (devido a problemas de alcoolismo, brigas entre o casal ou entre os filhos). Este percentual foi superior a $40,0 \%$ entre as adolescentes com renda familiar até 1,9 salários mínimos. Considerando a violência com parceiro sexual, $27,2 \%$ das adolescentes com parceiro sexual relataram algum tipo de violência, sendo que o menor percentual ocorreu entre as adolescentes de maior renda familiar $(18,8 \%)$. Em segundo lugar, percebe-se que $20,4 \%$ das adolescentes relataram violência familiar e violência com parceiro sexual, enquanto $56,6 \%$ não relataram nenhum tipo de violência, percentual este que variou de $53,8 \%$ a $58,9 \%$, da mais baixa até a mais alta categoria de renda.

$\mathrm{O}$ acesso às informações sobre sexualidade, contracepção, DST e AIDS e características do comportamento sexual das adolescentes estão descritos na Tabela 3. Apesar de mais de 90,0\% ter relatado acesso às informações sobre riscos e prevenção de DST/AIDS, a presença de história de DST ocorreu em 12,8\% da amostra. Destaca-se ainda que $31,6 \%$ já haviam engravidado, com $23,8 \%$ destas relatando aborto provocado. Apenas $23,4 \%$ das adolescentes relataram uso regular de preservativo em todas as relações sexuais, enquanto $34,7 \%$ declararam usarem raramente ou nunca.

Na Tabela 4, encontram-se os relatos sobre as percepções em relação ao uso do preservativo de acordo com o grau de escolaridade. $\mathrm{Ob}$ serva-se que cerca de $28,0 \%$ acha que não é fácil propor ao parceiro o uso de preservativo, independentemente do grau de escolaridade. Diferenças maiores por grau de instrução foram encontradas nas proporções de adolescentes que relataram que o pedido de uso de preservativo pelo parceiro demonstra falta de con-

Tabela 2

História de violência familiar e com parceiro sexual segundo a renda familiar das adolescentes residentes na região de Maruípe, Vitória, Espírito Santo, Brasil.

\begin{tabular}{|c|c|c|c|c|c|c|c|}
\hline \multirow[t]{3}{*}{ Renda familiar } & \multirow{3}{*}{$\begin{array}{l}\text { Relato de } \\
\text { violência } \\
\text { familiar }\end{array}$} & \multicolumn{4}{|c|}{$\begin{array}{l}\text { Relato de violência } \\
\text { com parceiro sexual }\end{array}$} & \multicolumn{2}{|c|}{ Total } \\
\hline & & \multicolumn{2}{|c|}{ Sim } & \multicolumn{2}{|c|}{ Não } & \multirow[b]{2}{*}{$\mathrm{n}$} & \multirow[b]{2}{*}{$\%$} \\
\hline & & $\mathrm{n}$ & $\%$ & $\mathrm{n}$ & $\%$ & & \\
\hline Até $\mathrm{R} \$ 399,00$ & Sim & 23 & 24,7 & 15 & 16,1 & 38 & 40,9 \\
\hline \multirow[t]{2}{*}{ (até 1,9 salários mínimos *) } & Não & 5 & 5,4 & 50 & 53,8 & 55 & 59,1 \\
\hline & Total & 28 & 30,1 & 65 & 69,9 & 93 & 100,0 \\
\hline De $\mathrm{R} \$ 400,00$ a $\mathrm{R} \$ 539,00$ & Sim & 25 & 21,2 & 16 & 13,6 & 41 & 34,7 \\
\hline \multirow[t]{2}{*}{ (de 2 a 2,6 salários mínimos) } & Não & 11 & 9,3 & 66 & 55,9 & 77 & 65,3 \\
\hline & Total & 36 & 30,5 & 82 & 69,5 & 118 & 100,0 \\
\hline De $R \$ 540,00$ a $R \$ 719,00$ & Sim & 20 & 22,5 & 11 & 12,4 & 31 & 34,8 \\
\hline \multirow[t]{2}{*}{ (de 2,7 a 3,6 salários mínimos) } & Não & 7 & 7,9 & 51 & 57,3 & 58 & 65,2 \\
\hline & Total & 27 & 30,3 & 62 & 69,7 & 89 & 100,0 \\
\hline $\mathrm{R} \$ 720,00$ a $\mathrm{R} \$ 3.000,00$ & Sim & 16 & 14,3 & 25 & 22,3 & 41 & 36,6 \\
\hline \multirow[t]{2}{*}{ (de 3,7 a 15 salários mínimos) } & Não & 5 & 4,5 & 66 & 58,9 & 71 & 63,4 \\
\hline & Total & 21 & 18,8 & 91 & 81,2 & 112 & 100,0 \\
\hline \multirow[t]{3}{*}{ Total } & Sim & 84 & 20,4 & 67 & 16,3 & 151 & 36,7 \\
\hline & Não & 28 & 6,8 & 233 & 56,6 & 261 & 63,3 \\
\hline & Total & 112 & 27,2 & 300 & 72,8 & 412 & 100,0 \\
\hline
\end{tabular}

* O salário mínimo na época do estudo era de $\mathrm{R} \$ 200,00$. 
fiança $(34,0 \%$ para ensino fundamental e 19,0\% para ensino médio).

Em relação ao uso de preservativo, tanto na primeira, na última relação sexual e na freqüência de uso, a proporção foi significativamente maior entre as de melhor escolaridade ( $\mathrm{p}<5,0 \%$ ). Da mesma forma, o pedido de uso pelo parceiro como o fato de querer usar também foram relativamente mais freqüentes entre as adolescentes de maior grau de instrução. Entretanto, independentemente do grau de escolaridade, proporção elevada foi encontrada (cerca de 45,0\%) de jovens que tiveram relação sexual sem preservativo, apesar de terem demonstrado vontade de usar.

Na Tabela 5, apresenta-se a associação entre o conhecimento sobre as formas de transmissão e o uso de preservativo na última relação sexual. Primeiramente, observa-se que a maior proporção de acertos foi relativa à transmissão por via sexual $(94,4 \%)$, enquanto a menor correspondeu à doação de sangue $(36,0 \%)$. Em segundo lugar, percebe-se que aquelas que já tiveram relação sexual mas não usaram preservativo na última relação têm, em geral, menor grau de conhecimento. Entre as entrevistadas, $11,0 \%$ responderam que namorariam uma pessoa infectada pelo HIV, 77,6\% têm conhecimento que há pessoas infectadas e que não sabem de sua situação, e aproximadamente, 46,0\% têm medo de se infectarem.

\section{Discussão}

No presente estudo, foram analisadas adolescentes do sexo feminino, na faixa etária de 15 a 19 anos, residentes em uma região de Vitória assistida pelo PSF, segundo o padrão de comportamento. A taxa de participação no estudo foi muito alta, quase $100,0 \%$.

Tendo em vista que o trabalho com adolescentes torna necessária a autorização dos pais pelo Código de Ética em Pesquisa 13, projetos que abordam a sexualidade enfrentam, freqüentemente, dificuldades pois não é fácil propor, discutir e garantir o sigilo às adolescentes. Este aspecto consumiu grande tempo do projeto (quatro meses aproximadamente) em reuniões com os pais e as adolescentes, em conjunto e em separado, para garantir a confidencialidade e a importância da adesão. Apesar do tempo consumido, a experiência foi proveitosa porque mostrou ser possível, com muito diálogo e negociação, conquistar a confiança das participantes, permitindo obter as informações das adolescentes com a mínima interferência dos pais.
Tabela 3

Comportamento sexual relatado pelas adolescentes residentes na região de Maruípe, Vitória, Espírito Santo, Brasil.

\begin{tabular}{|c|c|c|}
\hline Variáveis & $\mathbf{n}$ & $\%$ \\
\hline Acesso a informação sobre sexualidade & 421 & 90,7 \\
\hline Acesso a informação sobre contracepção & 437 & 94,2 \\
\hline Acesso a informação sobre DST & 426 & 91,8 \\
\hline Acesso a informação sobre AIDS & 429 & 92,5 \\
\hline Realização de exame ginecológico nos últimos dois anos & 207 & 44,6 \\
\hline Relato de relação sexual & 320 & 69,0 \\
\hline Relato de sexo vaginal & 319 & 68,8 \\
\hline Relato de sexo anal & 34 & 7,3 \\
\hline \multicolumn{3}{|l|}{ Freqüência de relação sexual no último mês } \\
\hline Nenhuma & 144 & 31,0 \\
\hline $1-4$ vezes & 117 & 25,2 \\
\hline $5-8$ vezes & 106 & 22,8 \\
\hline $9-12$ vezes & 60 & 12,9 \\
\hline 13 ou mais vezes & 37 & 8,1 \\
\hline Já realizou teste sorológico para HIV & 79 & 17,0 \\
\hline \multicolumn{3}{|l|}{ Uso regular de preservativos* } \\
\hline Sempre & 75 & 23,4 \\
\hline Às vezes & 134 & 41,9 \\
\hline Raramente & 66 & 20,6 \\
\hline Nunca & 45 & 14,1 \\
\hline Uso de contraceptivo oral* & 61 & 19,1 \\
\hline História de DST* & 41 & 12,8 \\
\hline Estupro* & 34 & 10,6 \\
\hline Prostituição* & 12 & 3,7 \\
\hline Gravidez* & 101 & 31,6 \\
\hline \multicolumn{3}{|l|}{ Desfecho da gravidez } \\
\hline Parto** & 69 & 68,3 \\
\hline Aborto espontâneo** & 18 & 17,8 \\
\hline Aborto provocado** & 24 & 23,8 \\
\hline
\end{tabular}

* Dados relacionados a 320 adolescentes que relataram atividade sexual.

** Dados relacionados a 101 adolescentes que relataram história de gravidez.

As características sócio-demográficas das adolescentes incluídas no estudo refletem as características de todas adolescentes assistidas pelo PSF em Maruípe, região de saúde do Município de Vitória que é constituída por bairros de classe média baixa e baixa mas com acesso a escolas e aos serviços públicos de saúde, e que, na sua maioria, têm grau médio de escolaridade.

As drogas lícitas e ilícitas investigadas foram o cigarro, o álcool, a maconha, os medicamentos psicotrópicos, o crack, a cocaína inala- 
Tabela 4

Percepções em relação ao uso do preservativo segundo escolaridade entre as adolescentes residentes na região de Maruípe, Vitória, Espírito Santo, Brasil.

\begin{tabular}{|c|c|c|c|}
\hline \multirow[t]{2}{*}{ Percepções em relação ao preservativo } & \multicolumn{2}{|c|}{ Grau de escolaridade } & \multirow[t]{2}{*}{ Total } \\
\hline & $\begin{array}{l}\text { Fundamental } \\
\quad(n=162)\end{array}$ & $\begin{array}{c}\text { Ensino médio/ } \\
\text { superior }(n=302)\end{array}$ & \\
\hline \multicolumn{4}{|l|}{ Em relação ao preservativo masculino, você acha que $(n=464)$} \\
\hline Não é fácil propô-lo ao parceiro & $47(29,0 \%)$ & $86(28,5 \%)$ & $133(28,7 \%)$ \\
\hline Demonstra falta de confiança & $55(34,0 \%)$ & $57(18,9 \%)$ & $112(24,1 \%)$ \\
\hline É engraçado & $37(22,8 \%)$ & $50(16,6 \%)$ & $87(18,8 \%)$ \\
\hline Atrapalha o prazer & $40(24,7 \%)$ & $61(20,2 \%)$ & $101(21,8 \%)$ \\
\hline Usou preservativo na primeira relação $(n=320)$ & $54(45,0 \%)$ & $122(61,0 \%)$ & $176(55,0 \%)$ \\
\hline Usou preservativo na última relação $(n=320)$ & $49(40,8 \%)$ & $119(59,5 \%)$ & $168(52,5 \%)$ \\
\hline Declarou sempre usar o preservativo & $23(19,2 \%)$ & $52(26,0 \%)$ & $75(23,4 \%)$ \\
\hline Querer usar preservativo com alguém que não queria $(n=320$ ) & $35(21,6 \%)$ & $72(23,8 \%)$ & $107(23,1 \%)$ \\
\hline \multicolumn{4}{|l|}{ Na última vez que isso aconteceu $(n=107)$} \\
\hline Vocês utilizaram o preservativo & $5(14,3 \%)$ & $18(25,0 \%)$ & $23(21,5 \%)$ \\
\hline Vocês tiveram relação sexual sem preservativo & $16(45,7 \%)$ & $32(44,4 \%)$ & $48(44,9 \%)$ \\
\hline Vocês não tiveram relação sexual & $13(37,1 \%)$ & $21(29,2 \%)$ & $34(31,8 \%)$ \\
\hline Vocês tiveram relação sexual sem penetração & $1(2,9 \%)$ & $1(1,4 \%)$ & $2(1,9 \%)$ \\
\hline
\end{tabular}

Tabela 5

Conhecimento sobre as formas de transmissão da AIDS segundo o uso de preservativo na última relação sexual entre as adolescentes residentes na região de Maruípe, Vitória, Espírito Santo, Brasil.

\begin{tabular}{|c|c|c|c|c|}
\hline \multirow[t]{3}{*}{ Formas de transmissão da AIDS } & \multicolumn{4}{|c|}{ Número e percentual de respostas certas } \\
\hline & \multicolumn{2}{|c|}{ Já tiveram relação sexual } & \multirow{2}{*}{$\begin{array}{l}\text { Não tiveram } \\
\text { relação sexual } \\
(n=144)\end{array}$} & \multirow{2}{*}{$\begin{array}{c}\text { Total } \\
(n=464)\end{array}$} \\
\hline & $\begin{array}{l}\text { Usou preservativo } \\
\qquad(n=176)\end{array}$ & $\begin{array}{l}\text { Não usou } \\
\text { preservativo } \\
(n=144)\end{array}$ & & \\
\hline 1. Transmite através da relação sexual & $172(97,7 \%)$ & $136(94,4 \%)$ & $139(96,5 \%)$ & $447(94,4 \%)$ \\
\hline 2. Não transmite em banheiros públicos & $123(69,9 \%)$ & $89(61,8 \%)$ & $100(69,4 \%)$ & $312(67,2 \%)$ \\
\hline 3. Através da partilha de seringas entre UDI & $167(94,9 \%)$ & $135(93,8 \%)$ & $135(93,8 \%)$ & $437(94,2 \%)$ \\
\hline 4. Através de transfusão sangüínea & $157(89,2 \%)$ & $124(86,1 \%)$ & $133(92,4 \%)$ & $414(89,2 \%)$ \\
\hline 5. Não transmite na doação de sangue & $61(34,7 \%)$ & $57(39,6 \%)$ & $49(34,0 \%)$ & $167(36,0 \%)$ \\
\hline 6. Não transmite através da picada de mosquito & $121(68,8 \%)$ & $94(65,3 \%)$ & $101(70,1 \%)$ & $316(68,1 \%)$ \\
\hline 7. Estar hospitalizado no mesmo quarto que uma pessoa com AIDS & $123(69,9 \%)$ & $87(60,4 \%)$ & $111(77,1 \%)$ & $321(69,2 \%)$ \\
\hline Percepções em relação à infecção pelo HIV & \multicolumn{3}{|c|}{ Número e percentual de opiniões relatadas } & Total \\
\hline 1. Você estudaria ou trabalharia com uma pessoa HIV+ & $165(93,8 \%)$ & $125(86,8 \%)$ & $136(94,4 \%)$ & $426(91,8 \%)$ \\
\hline 2. Você comeria na casa de uma pessoa HIV+ & $138(78,4 \%)$ & $106(73,6 \%)$ & $111(77,1 \%)$ & $355(76,5 \%)$ \\
\hline 3. Você namoraria uma pessoa HIV+ & $17(9,7 \%)$ & $19(13,2 \%)$ & $15(10,4 \%)$ & $51(11,0 \%)$ \\
\hline 4. Você conhece alguém com infecção pelo HIV & $51(29,0 \%)$ & $42(29,2 \%)$ & $47(32,6 \%)$ & $140(30,2 \%)$ \\
\hline 5. Você acha que há pessoas com infecção pelo HIV e que não sabem & $143(81,3 \%)$ & $105(72,9 \%)$ & $112(77,8 \%)$ & $360(77,6 \%)$ \\
\hline 6. Você tem medo de ser infectada pelo vírus HIV & $95(54,0 \%)$ & $69(47,9 \%)$ & $48(33,3 \%)$ & $212(45,7 \%)$ \\
\hline
\end{tabular}


tória e drogas injetáveis. A entrevista foi face a face, o que pode ter gerado uma subestimação dos resultados devido ao preconceito e ao temor das adolescentes em responder questões nesta área do comportamento. Adicionalmente, a amostra tem a peculiaridade de ter grau de escolaridade e renda familiar média superior à da população brasileira. Mas, feitas estas ressalvas, os resultados obtidos demonstraram estar em concordância com os dados resultantes da pesquisa sobre Comportamento Sexual da População Brasileira e Percepções do HIVIAIDS 10 onde $16,3 \%$ dos adolescentes, de 16 a 19 anos, declararam já ter utilizado algum tipo de droga. Da mesma forma, dados do último levantamento realizado pelo Centro Brasileiro de Informações sobre Drogas Psicotrópicas (CEBRID) entre crianças e adolescentes, escolarizados em 1997, relatam que as drogas lícitas e ilícitas são usadas rotineiramente por $15,0 \%$ dos escolares 14. No presente estudo, o percentual encontrado foi de $14,0 \%$.

A história de violência - familiar ou causada pelo parceiro sexual - foi relatada freqüentemente neste estudo: $33,8 \%$ relataram alguma forma de violência familiar e $27,2 \%$ violência por parte do parceiro sexual. Um dado relevante foi a associação entre violência familiar e violência com o parceiro, já que, entre as que relataram algum tipo de violência familiar, a violência provocada pelo parceiro era mais prevalente.

Os efeitos da violência doméstica, sexual e racial, entre as mulheres, sobre a saúde física e mental têm sido evidenciados em outros trabalhos (Rufino A; 1999, comunicação pessoal). Em relação às crianças e adolescentes, Roque \& Ferriani 15 apresentaram um estudo sobre a violência, conduzido no Fórum da cidade de Jardinópolis em São Paulo, com análise sob o ponto de vista jurídico. Os resultados mostraram que a violência doméstica está associada com a falta de estrutura familiar, condições econômicas e sociais precárias, casamentos instáveis, problemas mentais, alcoolismo e a falta de políticas sociais para controlar a situação.

Neste estudo, 69,0\% das participantes relataram já ter iniciado atividade sexual, mas somente $23,0 \%$ faziam uso regular de preservativo. Apesar do acesso às informações sobre sexualidade, contracepção e DST/AIDS, a proporção de uso regular de preservativos foi baixa e as proporções de história de gravidez e DST foram elevadas. Estes dados estão em concordância com os resultados da pesquisa realizada pelo Centro Brasileiro de Análise e Planejamento (CEBRAP) que indicam que, atualmente, os adolescentes têm a primeira relação se- xual mais precocemente que as gerações anteriores, mas os níveis de conhecimento sobre as DST/AIDS não alteram o comportamento sexual de risco.

O presente estudo mostrou o papel do grau de escolaridade como variável explicativa das diferenças referentes às práticas sexuais de risco das adolescentes do sexo feminino. As jovens com maior grau de escolaridade relataram uso mais freqüente de preservativo, seja na última ou na primeira relação sexual, ou quando se trata de uso regular. Estes resultados foram semelhantes aos encontrados em estudo com adolescentes do sexo masculino, mediante pesquisa com conscritos do Exército do Brasil. Os jovens com primeiro grau incompleto iniciam mais precocemente o relacionamento sexual, têm uma freqüência maior de atividade sexual, um número maior de parceiros casuais, uma menor freqüência de utilização do preservativo, o que implica em piores índices de comportamento sexual de risco 16. Estudos realizados nos Estados Unidos mostram também que, neste país, a utilização do preservativo entre as adolescentes não é uma prática corrente apesar do conhecimento sobre o método e sobre sua importância na prevenção das DST 17,18. Dados de estudos europeus, por outro lado, mostram freqüência bem maior de uso de preservativo. Estudo realizado em Nantes (França) mostrou que $75,0 \%$ das jovens que participaram no estudo utilizaram algum método contraceptivo durante a primeira relação sexual e que o preservativo foi o método mais usado para evitar contracepção 19 .

Neste estudo, foi encontrado alto percentual de gravidez na adolescência $(32,0 \%)$ e, apesar da ilegalidade na lei brasileira, uma alta freqüência de aborto provocado. A Pesquisa Nacional de Demografia e Saúde (PNDS) 20, realizada em 1996, encontrou uma proporção menos elevada (18,0\%) de adolescentes que já haviam engravidado pelo menos uma vez.

Em relação às percepções entre o uso do preservativo e o conhecimento sobre as formas de transmissão do vírus da AIDS, foi observado que, independentemente do grau de escolaridade, do conhecimento sobre as formas de transmissão e da percepção quanto à susceptibilidade à infecção, ainda há ressalvas quanto ao uso do preservativo de forma generalizada. Apesar do maior percentual de adolescentes que relatou uso de preservativo na primeira e na última relação sexual entre as jovens com maior instrução, não houve diferença entre os dois grupos, quando a falta de uso foi motivada pela recusa do parceiro. Quando são observadas as respostas obtidas sobre as formas de 
transmissão da AIDS, percebe-se que o percentual de acerto é alto em relação à transmissão sexual $(94,4 \%)$, ao compartilhamento de seringas entre UDI $(94,2 \%)$ e à transfusão de sangue $(89,2 \%)$ mas baixo em relação à doação de sangue $(36,0 \%)$, categoria esta que ainda gera muitas dúvidas no momento da resposta. Estes dados estão de acordo com os resultados da pesquisa com jovens do Exército Brasileiro em 1997, que também encontrou um alto percentual de acertos em relação à transmissão sangüínea e um baixo percentual em relação à doação de sangue 21, e com a PNDS 22, que também encontrou resultados semelhantes. Estes dados demonstram que, apesar do conhecimento sobre as formas de transmissão do HIV não ser suficiente para alterar significativamente o comportamento de risco, as estratégias para a ampliação do conhecimento relacionado ao HIV/ AIDS não devem ser negligenciadas, uma vez que este seria o primeiro passo na direção da percepção correta do risco à infecção pelo HIV.

Os resultados aqui encontrados mostram a importância de implementação de serviços de aconselhamento e assistência à saúde reprodutiva, que sejam dirigidos, especificamente, a este subgrupo populacional. Apesar da urgente necessidade de melhorar o nível de consciência pública, as barreiras culturais e institucionais dificultam programas de educação dos adolescentes em relação ao comportamento sexual de risco bem como quanto ao uso de drogas 22 . Alguns pais e educadores ainda expressam sua preocupação sobre a possibilidade das informações desses assuntos estimularem a atividade sexual entre os adolescentes. Entretanto, um estudo realizado pelo Programa das Nações Unidas sobre HIV/AIDS 23 mostrou que atividades educativas sobre HIV/AIDS e saúde sexual promovem condutas sexuais mais saudáveis sem aumentar o índice de atividade se- xual, além de proteger contra as doenças sexualmente transmissíveis.

Os programas educacionais direcionados aos adolescentes devem incluir alguns elementos-chave para obterem êxito: uma programação planejada, controle regular, abordagem de questões específicas deste grupo populacional e prevenção e assistência às infecções sexualmente transmissíveis, incluindo o HIV. Os programas devem ser abrangentes, compreendendo tanto os adolescentes como os pais e educadores 5,7 .

A prevenção da infecção pelo HIV e de outras DST baseada no desenvolvimento de estratégias de redução do número de parceiros e no incentivo a relacionamentos responsáveis tem se mostrado eficaz para adolescentes 24 . Como evidenciado neste estudo, o conhecimento sobre os meios de transmissão das DST e dos métodos contraceptivos não é suficiente para ajudar na proteção; os adolescentes precisam aprender a identificar uma situação de risco, compreender sua vulnerabilidade, conhecer as alternativas que eles possuem para se proteger, decidir qual alternativa é melhor para cada situação e para seus valores pessoais, diante da conscientização do risco e dimensionamento das conseqüências posteriores 7,25.

Outro aspecto importante a ser enfatizado é que a introdução de métodos mais eficazes de abordagem das DST não será, em si, eficaz no controle dessas doenças, caso os adolescentes continuem sem acesso aos medicamentos e ao apoio prático e emocional necessários para a prevenção e o tratamento. Tendo em vista que esta população é assistida pelo PSF, é necessário estabelecer estratégias conjuntas com a participação da família, dos educadores e dos profissionais de saúde para obter maior êxito no controle das DST. 


\section{Resumo}

Adolescentes constituem um subgrupo populacional vulnerável às doenças sexualmente transmissíveis (DST). O objetivo foi descrever o padrão de comportamento de adolescentes do sexo feminino, de 15 a 19 anos, relacionado às práticas sexuais e uso de drogas, residentes na região de Maruípe em Vitória, Brasil, assistida pelo Programa Saúde da Família. Foi realizado um estudo descritivo, de março a junho de 2002, aplicadas entrevistas face a face e coletada uma amostra de urina para realização de teste para Chlamydia trachomatis. Durante o estudo, 464 adolescentes foram incluídas, 69,0\% das adolescentes já tinham iniciado vida sexual; 12,8\% relataram história de DST; 14,0\% o uso de alguma droga ilícita e 3,7\% história de prostituição. Somente 23,4\% relataram uso regular de preservativos apesar de mais de 90,0\% ter relatado acesso às informações sobre riscos e prevenção de DST/AIDS. A história de gravidez foi relatada por $31,6 \%$ das adolescentes e a realização prévia do teste HIV foi relatada por 17,0\%. Apesar de terem conhecimento das formas de transmissão das DST/AIDS, as adolescentes não se previnem adequadamente. Os resultados mostram a necessidade de ações preventivas, incluindo, entre outras, testes de rotina para detecção de DST e programas de redução de riscos.

Adolescente; Doenças Sexualmente Transmissíveis; Síndrome de Imunodeficiência Adquirida; Comportamento Sexual

\section{Colaboradores}

A. E. Miranda contribuiu para a concepção, planejamento, análise e interpretação dos dados; na elaboração do rascunho e na revisão crítica do conteúdo; e da aprovação da versão final do manuscrito. A. J. Gadelha contribuiu para a concepção e planejamento dos dados e na elaboração do rascunho do manuscrito. C. L. Szwarcwald contribuiu para a análise e interpretação dos dados; na elaboração do rascunho e na revisão crítica do conteúdo; e da aprovação da versão final do manuscrito.

\section{Agradecimentos}

Agradecemos ao Fundo de Apoio à Ciência e Tecnologia do Município de Vitória (FACITEC) e ao Ministério da Saúde (PN-DST/AIDS - UNESCO: Projeto 914 BRA, 5o termo aditivo acordo Brasil-França) pelo suporte financeiro.

\section{Referências}

1. Landry DJ, Singh S, Darroch JE. Sexuality and education in fifth and sixth grades in US public schools, 1999. Fam Plann Perspect 2000; 35:212-9.

2. World Health Organization. Aider les jeunes a faire des choix sains en matiere de sexualité et de procriation, declare le directeur general de l'OMS. Geneva: World Health Organization; 1999.

3. Brasil. Estatuto da criança e do adolescente. Lei no 8.069, de 13 de julho de 1990. Diário Oficial da União 1990; 16 jul.

4. Comissão Nacional de População e Desenvolvimento. Jovens acontecendo na trilha das políticas públicas. Brasília: Comissão Nacional de População e Desenvolvimento; 1998.

5. Population Reference Bureau. La juventud del mundo 2000. Washington DC: Population Reference Bureau; 2000.

6. Aquino EML, Araújo TVB, Marinho LFB. Padrões e tendências em saúde reprodutiva no Brasil: bases para uma análise epidemiológica. In: Giffin K, Costa SH, organizadores. Questões da saúde reprodutiva. Rio de Janeiro: Editora Fiocruz; 1999. p. 187-204.

7. Gevelber MA, Biro F. Adolescents and sexually transmitted diseases. Pediatr Clin North Am 1999; 46:747-66.

8. Wasserheit JN. The significance and scope of reproductive tract infections among third world women. Suppl Int J Gynaecol Obstet 1989; 3:145-68.

9. Wasserheit JN. Reproductive tract infections: global impact and priorities for women's reproductive health. New York: Plenum Press; 1998.

10. Centro Brasileiro de Análise e Planejamento. Comportamento sexual da população brasileira e percepções sobre HIV e AIDS. Brasília: Ministério da Saúde; 2000.

11. Lagrange $\mathrm{H}$, Lhomond B. L'entrée dans la sexualité: le comportement des jeunes dans le contexte du SIDA. Paris: La Découvert; 1997.

12. Ministério da Saúde. Manual de controle das doenças sexualmente transmissíveis. Brasília: Coordenação de Doenças Sexualmente Transmissíveis e AIDS, Secretaria de Projetos Especiais de Saúde, Ministério da Saúde; 1999.

13. Conselho Nacional de Saúde. Resolução no 196, de 10 de outubro de 1996. http://www.datasus. gov.br/conselho/resol96/RES19696.htm (acessado em 05/Dez/2003).

14. Coordenação de Doenças Sexualmente Transmissíveis e AIDS, Secretaria de Projetos Especiais de Saúde, Ministério da Saúde. A vulnerabilidade de crianças e adolescentes. http://www.aids.gov.br/ drogas/sexualidade/sexualidade_prefacio.htm (acessado em 20/Dez/2003).

15. Roque EM, Ferriani MG. Unveiling domestic violence against children and adolescents under the point of the legal professionals in the municipality of Jardinópolis, São Paulo, Brazil. Rev Lat Am Enfermagem 2002; 10:334-44.

16. Szwarcwald CL, Castilho EA, Barbosa AJ, Gomes MRO, Costa EAMM, Maletta BV, et al. Comportamento de risco dos conscritos do Exército Brasileiro, 1998: uma apreciação da infecção pelo HIV segundo diferenciais sócio-econômicos. Cad Saú- 
de Pública 2000; 16 Suppl 1:113-28.

17. Roye CF. Condom use by Hispanic and AfricanAmerican adolescent girls who use hormonal contraception. J Adolesc Health 1998; 24:205-11.

18. Shrier LA, Goodman E, Emans SJ. Partner condom use among adolescent girls with sexually transmitted diseases. J Adolesc Health 1999; 23: 357-61.

19. Lemort MF, Lemort JP, Lopes P. Contraception during the first sexual intercourse: a survey concerning 467 female teenagers, 13 to 21 years in the Nantes area. Contracept Fertil Sex (Paris) 1999; 27:197-202.

20. Sociedade Civil Bem-Estar Familiar no Brasil. Brasil: Pesquisa Nacional sobre Demografia e Saúde: uma análise do nível de conhecimento e comportamentos de vulnerabilização. Rio de Janeiro: Sociedade Civil Bem-Estar Familiar no Brasil; 1997.

21. Ministério da Saúde. Pesquisa entre os conscritos do Exército Brasileiro, 1996-2000: retratos do comportamento de risco do jovem brasileiro à infecção pelo HIV. Brasília: Coordenação de Doenças Sexualmente Transmissíveis e AIDS, Secretaria de Projetos Especiais de Saúde, Ministério da Saúde; 2002.
22. Ruzany MH, Peres EM, Meirelles ZJ, Calazans ML, Grossman E, Kuschnir MC, et al. Multidisciplinary training program in the field of integral care for adolescents. Educ Med Salud 1995; 29:112-8.

23. Joint United Nations Programme on HIV/AIDS. Impact of HIV and sexual health education on the sexual behaviour of young people: a review update. Geneva: Joint United Nations Programme on HIV/AIDS; 1997.

24. Youssef M, Wallace B, Connoly M, Franzkowiak P. Working with young people: a guide to preventing HIV/AIDS and STDs. London: Commonwealth Youth Programme; 1995.

25. Lagarde E, Pison G, Enel C. A study of sexual behavior change in rural Senegal. J Acquir Immune Defic Syndr Hum Retrovirol 1996; 11:282-7.

Recebido em 02/Dez/2003

Versão final reapresentada em 15/Jun/2004 Aprovado em 10/Ago/2004 